\title{
Efficacy of continuous intravenous pumping of insulin for patients with diabetes complicated with perianal abscess and the effect on inflammatory cytokines
}

\author{
ZHIFENG ZHONG ${ }^{1}$, HUAYING HUANG ${ }^{2}$, YUEJUN HAN ${ }^{1}$ and WUZHEN DONG ${ }^{1}$ \\ Departments of ${ }^{1}$ Colorectal and Anal Surgery, and ${ }^{2}$ Endocrinology and Metabolism, \\ Jinhua Hospital of Zhejiang University, Jinhua, Zhejiang 321000, P.R. China
}

Received April 15, 2019; Accepted June 10, 2019

DOI: $10.3892 /$ etm.2019.7773

\begin{abstract}
Efficacy of insulin with different administrations for patients with diabetes complicated with perianal abscess and the effect on serum inflammatory cytokines were investigated. One hundred and sixty-seven patients with type 2 diabetes who underwent radical operation of perianal abscess in Jinhua Hospital of Zhejiang University from January 2014 to December 2016 were analyzed. Before and after the operation, 89 patients who received continuous intravenous pumping of insulin for blood glucose control were set as an observation group, and 78 patients who received intermittent subcutaneous injection of insulin as a control group. The operative efficacy, wound healing time and 1-week postoperative growth of the granulation tissue were scored and compared. Fasting blood glucose (FBG) and $2 \mathrm{~h}$ postprandial blood glucose (2hPBG) before and after treatment were recorded and compared. Fasting venous blood was extracted before and on the 3rd and 7th days after operation to detect and compare serum inflammatory cytokines including tumor necrosis factor (TNF- $\alpha$ ) and interleukin-6 (IL-6). Patients in the observation group had significantly higher total effective rate of the operation than that in the control group $(\mathrm{P}<0.05)$, and significantly shorter wound healing time and significantly lower growth score of the granulation tissue $(\mathrm{P}<0.05)$. Before treatment, there was no significant difference between the two groups in FBG and $2 \mathrm{hPBG}(\mathrm{P}>0.05)$. After treatment, FBG and $2 \mathrm{hPBG}$ were significantly lower than those before treatment $(\mathrm{P}<0.050)$, and $\mathrm{FBG}$ and $2 \mathrm{hPBG}$ after treatment in the observation group were significantly lower than those in the control group $(\mathrm{P}<0.05)$. In conclusion, insulin pumps
\end{abstract}

Correspondence to: Dr Zhifeng Zhong, Department of Colorectal and Anal Surgery, Jinhua Hospital of Zhejiang University, 351 Mingyue Street, Wucheng, Jinhua, Zhejiang 321000, P.R. China E-mail: ief64d@163.com; zzf1373@163.com

Key words: diabetes complicated with perianal abscess, continuous intravenous pumping of insulin, intermittent subcutaneous injection of insulin, TNF- $\alpha$, IL-6, efficacy for injection during the perioperative period of patients with diabetes complicated with perianal abscess can better control the patients' blood glucose, improve the operative efficacy and promote the patients' postoperative healing. Moreover, continuous intravenous pumping of insulin is significantly better than traditional intermittent subcutaneous injection of it in controlling inflammation, so it is worthy of application.

\section{Introduction}

Diabetes is a common chronic disease in clinic. Pancreatic dysfunction leads to hypoinsulinism in the body, which increases sugar in blood and urine and eventually results in diabetes and its complications $(1,2)$. As one of the common complications (3), perianal abscess is a surgical disease caused by acute pyogenic infection in the soft tissue and space around the anal canal or the rectum. Generally speaking, operation is the most effective way to treat perianal abscess, but their large wound surface usually causes long postoperative recovery time $(4,5)$. Patients with diabetes who are in a hyperglycemic state for a long time have poorer ability of anti-infection and resistance than normal people, and surgical trauma easily leads to their reduction of insulin secretion. As a result, the patients develop metabolic disorders, which increase the difficulty and risk of a surgical operation and seriously affect the postoperative healing of wound surface, not conducive to the patients' prognosis $(6,7)$.

Therefore, it is imperative to control the blood glucose of patients with diabetes complicated with perianal abscess in order to improve the operative efficacy and reduce the risk of infections. The blood glucose of patients with diabetes is controlled through insulin, which can be orally administered, intravenously pumped or subcutaneously injected. Findings have shown that different administrations of insulin have different efficacy and safety (8). However, the efficacy of the different administrations for patients with diabetes complicated with perianal abscess has rarely been studied. According to a study on the wound surface healing of perianal abscess, tumor necrosis factor- $\alpha$ (TNF- $\alpha$ ) and interleukin-6 (IL-6) are representative inflammatory cytokines and they play an important role during wound surface healing (9). Therefore, the efficacy of insulin with different administrations for 
patients with diabetes complicated with perianal abscess and its effects on the postoperative healing of wound surface and serum inflammatory cytokines were explored in this study, in order to provide a more appropriate scheme for the treatment of the patients.

\section{Materials and methods}

General information. In total, 167 patients with type 2 diabetes who underwent radical operation of perianal abscess in Jinhua Hospital of Zhejiang University (Jinhua, China) from January 2014 to December 2016 were retrospectively analyzed. There were 91 males and 76 females, with an average age of $54.31 \pm 4.65$ years and an average course of diabetes of $4.31 \pm 1.29$ years. Before and after the operation, 89 patients who received continuous intravenous pumping of insulin for blood glucose control were set as the observation group, and 78 patients who received intermittent subcutaneous injection of insulin as the control group. Both groups of patients received routine anti-infection treatment after the radical operation. There was no significant difference in sex and age between the two groups of patients $(\mathrm{P}>0.05)$, which were comparable (Table I).

Inclusion and exclusion criteria. Inclusion criteria were: Patients diagnosed with diabetes complicated with perianal abscess. Exclusion criteria were: Patients with severe infectious diseases; patients with surgical contraindications; patients with other severe organ diseases or complicated with tumors; patients with coagulation disorders; patients with communication disorders; patients who did not cooperate with the study. The study was approved by the Ethics Committee of Jinhua Hospital of Zhejiang University. Patients who participated in this research had complete clinical data. The signed informed consents were obtained from the patients or the guardians.

Treatment methods. Patients in the control group were intermittently and subcutaneously injected with insulin for perioperative blood glucose control. Insulin was daily injected before meals, and its dose was adjusted according to the patients' blood glucose changes. Patients in the observation group were continuously pumped with insulin for $24 \mathrm{~h}$. The basic dose of the insulin pump was controlled at $50 \%$ of the standard dose, and the other $50 \%$ was additionally pumped before meals. The patients' blood glucose was monitored in real time. Perioperative anti-infection treatment and alimentary control were carried out on both groups of patients. The control range of preoperative blood glucose was as follows: fasting blood glucose $(\mathrm{FBG}) \leq 8.0 \mathrm{mmol} / \mathrm{l}$ and $2 \mathrm{~h}$ postprandial blood glucose $(2 \mathrm{hPBG}) \leq 11.0 \mathrm{mmol} / \mathrm{l}$. After the blood glucose was controlled, the operation was performed.

Observational indexes. i) The operative efficacy was scored and compared between the two groups. Markedly effective indicated normal defecation, normal blood glucose and wound surface healing after operation. Effective indicated slight pain during defecation, normal blood glucose and wound surface healing after operation. Invalid indicated blood glucose which was not significantly improved, strong pain during defecation and wound surface which did not heal after operation.
The total effective rate was calculated as: (markedly effective + effective cases) / total number of cases $\times 100 \%$. ii) The wound healing time and 1 week postoperative growth of the granulation tissue were scored and compared between the two groups. Scoring standards for the growth of the granulation tissue were as follows (10): 0 for good growth and wound surface healing; 1 point for vigorous growth with bright red and granular wound surface; 2 points for vigorous growth with light red and flat wound surface; 3 points for slow growth with murky grey and depressed wound surface. The lower the score, the better the healing was. iii) FBG and $2 \mathrm{hPBG}$ before and after treatment were recorded and compared between the two groups. iv) Fasting venous blood was extracted before and on the 3 rd and 7th days after operation to detect and compare serum inflammatory cytokines (TNF- $\alpha$ and IL-6) between the two groups using ELISA. ELISA kits were purchased from Shanghai Enzyme-linked Biotechnology Co., Ltd.

Statistical analysis. In this study, SPSS19.0 (IBM Corp. ) was used to statistically analyze the data. Chi-square test was used for comparison of enumeration data, t-test for comparison of measurement data, repeated measures analysis of variance with LSD post hoc test for comparison at multiple time points, and GraphPad Prism 6 for plotting figures. $\mathrm{P}<0.05$ was considered to indicate a statistically significant difference.

\section{Results}

Comparison of operative efficacy. There were 69 markedly effective cases, 15 effective cases and 5 invalid cases in the observation group, with a total effective rate of $94.38 \%$. There were 43 markedly effective cases, 16 effective cases and 19 invalid cases in the control group, with a total effective rate of $75.64 \%$. The total effective rate in the observation group was significantly higher than that in the control group $(\mathrm{P}<0.05)$ (Table II).

Wound healing time and 1-week postoperative growth score of the granulation tissue. The wound healing time and 1-week postoperative growth score of the granulation tissue in the observation group were $16.31 \pm 2.29$ days and $1.19 \pm 0.35$ points, while those in the control group were $22.97 \pm 3.75$ days and $2.09 \pm 0.36$ points. Compared with the control group, patients in the observation group had significantly shorter wound healing time and significantly lower growth score $(\mathrm{P}<0.05)$ (Table III).

$F B G$ and $2 h P B G$ before and after treatment. In the observation group, FBG was $10.31 \pm 2.65 \mathrm{mmol} / 1$ before treatment and $5.21 \pm 1.35 \mathrm{mmol} / 1$ after treatment, and $2 \mathrm{hPBG}$ was $12.65 \pm 2.26 \mathrm{mmol} / \mathrm{l}$ before treatment and $7.41 \pm 1.73 \mathrm{mmol} / \mathrm{l}$ after treatment. In the control group, FBG was $10.32 \pm 3.59 \mathrm{mmol} / 1$ before treatment and $6.59 \pm 1.76 \mathrm{mmol} / 1$ after treatment, and $2 \mathrm{hPBG}$ was $12.58 \pm 2.15 \mathrm{mmol} / \mathrm{l}$ before treatment and $8.83 \pm 2.55 \mathrm{mmol} / \mathrm{l}$ after treatment. Before treatment, there was no significant difference between the two groups in FBG and 2hPBG ( $\mathrm{P}>0.05)$. After treatment, FBG and $2 \mathrm{hPBG}$ in the two groups were significantly lower than those before treatment $(\mathrm{P}<0.05)$, and FBG and 2 hPBG after treatment in the observation group were significantly lower than those in the control group $(\mathrm{P}<0.05)$ (Figs. 1 and 2$)$. 
Table I. General information.

\begin{tabular}{|c|c|c|c|c|}
\hline Factors & Observation group $(n=89)$ & Control group $(n=78)$ & $\mathrm{t} / \chi^{2}$ value & P-value \\
\hline Sex & & & 0.024 & 0.877 \\
\hline Male & $48(53.93)$ & $43(55.13)$ & & \\
\hline Female & $41(46.07)$ & $35(44.87)$ & & \\
\hline Age (years) & & & 0.001 & 0.976 \\
\hline$\geq 54$ & $50(56.18)$ & $44(56.41)$ & & \\
\hline$<54$ & $39(43.82)$ & $34(43.59)$ & & \\
\hline BMI $\left(\mathrm{kg} / \mathrm{m}^{2}\right)$ & & & 0.013 & 0.910 \\
\hline$\geq 23$ & $43(48.31)$ & $37(47.44)$ & & \\
\hline$<23$ & $46(51.69)$ & $41(52.56)$ & & \\
\hline Types of abscess & & & 0.020 & 0.990 \\
\hline Retrorectal space abscess & $34(38.21)$ & $29(37.18)$ & & \\
\hline Submucosal abscess of rectum & $26(29.21)$ & $23(29.49)$ & & \\
\hline Pelvirectal abscess & $29(32.58)$ & $26(33.33)$ & & \\
\hline Course of diabetes (years) & $4.30 \pm 1.28$ & $4.32 \pm 1.31$ & 0.100 & 0.921 \\
\hline \multicolumn{5}{|l|}{ Coagulation function } \\
\hline APTT (sec) & $27.45 \pm 2.72$ & $28.01 \pm 2.61$ & 1.353 & 0.178 \\
\hline $\mathrm{PT}(\mathrm{sec})$ & $12.02 \pm 1.03$ & $12.05 \pm 1.05$ & 0.186 & 1.853 \\
\hline FIB (g/l) & $3.13 \pm 0.19$ & $3.12 \pm 0.24$ & 0.300 & 0.764 \\
\hline TT (sec) & $14.56 \pm 1.17$ & $14.51 \pm 1.21$ & 0.271 & 0.787 \\
\hline \multicolumn{5}{|l|}{ Liver function indexes } \\
\hline Total serum protein $(\mathrm{g} / \mathrm{l})$ & $70.19 \pm 2.75$ & $70.76 \pm 2.76$ & 1.334 & 0.184 \\
\hline Alanine aminotransferase $(\mu \mathrm{mol} / \mathrm{l})$ & $28.19 \pm 4.31$ & $27.92 \pm 4.17$ & 0.410 & 0.682 \\
\hline Total bilirubin $(\mu \mathrm{mol} / 1)$ & $11.28 \pm 2.16$ & $11.27 \pm 2.22$ & 0.029 & 0.977 \\
\hline \multicolumn{5}{|l|}{ Renal function indexes $(\mu \mathrm{mol} / \mathrm{l})$} \\
\hline Creatinine & $69.15 \pm 4.41$ & $70.05 \pm 4.31$ & 1.330 & 0.185 \\
\hline Serum urea & $5.33 \pm 0.87$ & $5.41 \pm 0.94$ & 0.571 & 0.569 \\
\hline Uric acid & $278.55 \pm 11.75$ & $281.31 \pm 11.93$ & 1.504 & 0.135 \\
\hline
\end{tabular}

Table II. Comparison of operative efficacy.

\begin{tabular}{lccr}
\hline Efficacy & Observation group $(\mathrm{n}=89)$ & Control group $(\mathrm{n}=78)$ & $\chi^{2}$ value \\
\hline Markedly effective & $69(77.53)$ & $43(55.13)$ & - \\
Effective & $15(16.85)$ & $16(20.51)$ & - \\
Invalid & $5(5.62)$ & $19(24.36)$ & - \\
Total effective rate & $84(94.38)$ & $59(75.64)$ & - \\
\hline
\end{tabular}

Table III. Wound healing time and 1-week postoperative growth score of the granulation tissue.

\begin{tabular}{|c|c|c|c|c|}
\hline Items & Observation group $(\mathrm{n}=89)$ & Control group $(n=78)$ & $\mathrm{t}$ value & P-value \\
\hline Wound healing time (days) & $16.31 \pm 2.29$ & $22.97 \pm 3.75$ & 14.04 & $<0.001$ \\
\hline Growth score of the granulation tissue & $1.19 \pm 0.35$ & $2.09 \pm \quad 0.36$ & 16.36 & $<0.001$ \\
\hline
\end{tabular}

Expression levels of TNF- $\alpha$ and IL-6 before and on the $3 r d$ and 7 th days after operation. In the observation group, the expression levels of TNF- $\alpha$ before and on the 3rd and 7 th days after operation were $195.24 \pm 23.52,101.23 \pm 15.39$ and $89.31 \pm 14.85 \mathrm{ng} / \mathrm{l}$, and the expression levels of IL-6 were $214.25 \pm 74.52,144.65 \pm 44.51$ and $87.82 \pm 18.29 \mathrm{ng} / 1$. In the control group, the expression levels of $\mathrm{TNF}-\alpha$ were $196.21 \pm 24.55,140.32 \pm 17.03$ and $121.07 \pm 25.67 \mathrm{ng} / \mathrm{l}$, and the 


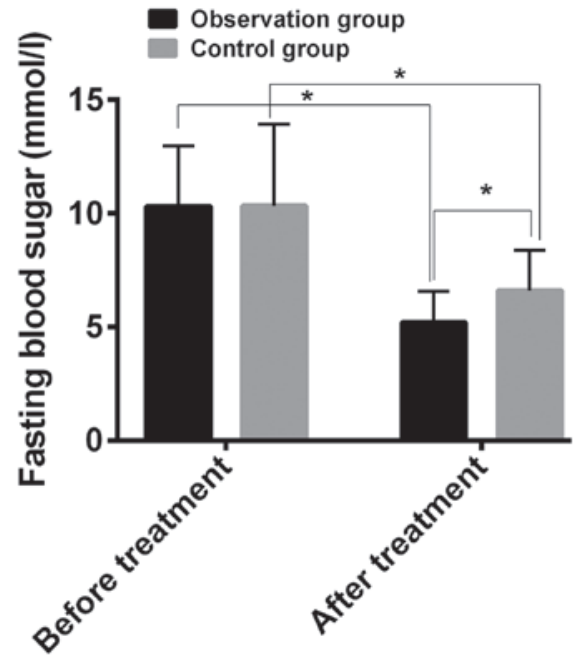

Figure 1. Comparison of FBG before and after treatment. Before treatment, there was no significant difference between the observation and control groups in FBG $(\mathrm{P}>0.05)$. After treatment, $\mathrm{FBG}$ in the observation group was significantly lower than that in the control group ( $\mathrm{P}<0.05)$.

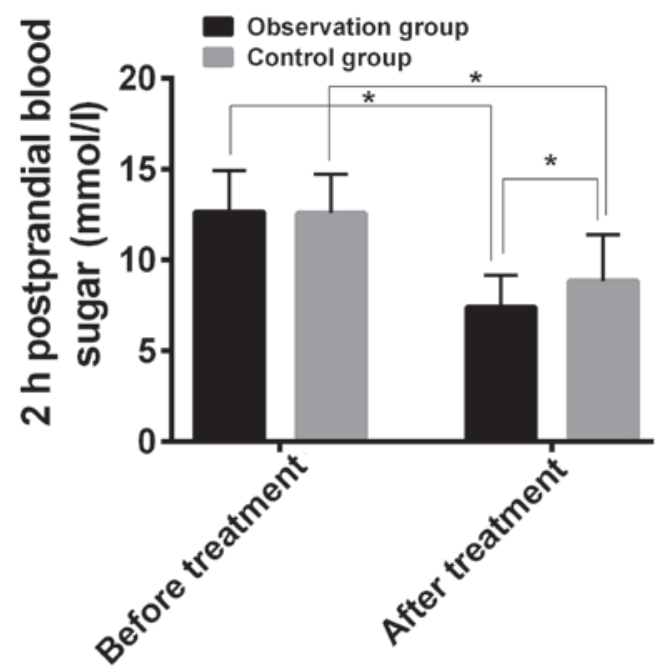

Figure 2. Comparison of $2 \mathrm{hPBG}$ before and after treatment. Before treatment, there was no significant difference between the observation and control groups in $2 \mathrm{hPBG}(\mathrm{P}>0.05)$. After treatment, $2 \mathrm{hPBG}$ in the observation group was significantly lower than that in the control group ( $(\mathrm{P}<0.05)$.

expression levels of IL-6 were $215.65 \pm 77.29,170.27 \pm 49.18$ and $120.29 \pm 28.05 \mathrm{ng} / \mathrm{l}$. Before treatment, there was no significant difference between the two groups in the expression levels of TNF- $\alpha$ and IL-6 ( $>>0.05)$. On the 3rd and 7th days after operation, the expression levels in the two groups were significantly lower than those before treatment, and the expression levels on the 7th day after treatment were significantly lower than those on the 3rd day after operation $(\mathrm{P}<0.05)$. On the $3 \mathrm{rd}$ and 7 th days after operation, the expression levels in the observation group were significantly lower than those in the control group $(\mathrm{P}<0.05)$ (Figs. 3 and 4$)$.

\section{Discussion}

The incidence of diabetes has been increasing with social progress and changes in diet structure in recent years (11).

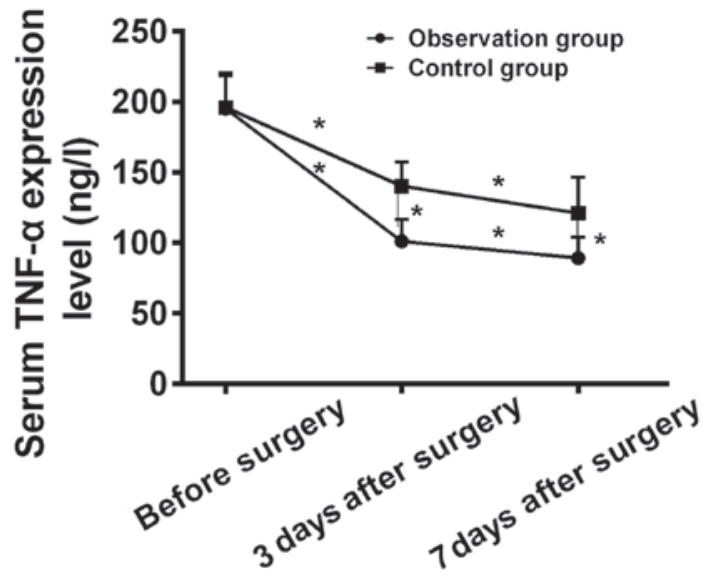

Figure 3. Expression level of TNF- $\alpha$ before and on the 3rd and 7th days after operation. According to ELISA, before treatment, there was no significant difference between the observation and control groups in the expression level of TNF- $\alpha(\mathrm{P}>0.05)$. On the $3 \mathrm{rd}$ and 7 th days after operation, the expression levels in the two groups were significantly lower than those before treatment, and the expression level on the 7 th day after treatment was significantly lower than that on the 3rd day after operation $\left({ }^{*} \mathrm{P}<0.05\right)$. On the $3 \mathrm{rd}$ and 7 th days after operation, the expression levels in the observation group were significantly lower than those in the control group $\left({ }^{*} \mathrm{P}<0.05\right)$.

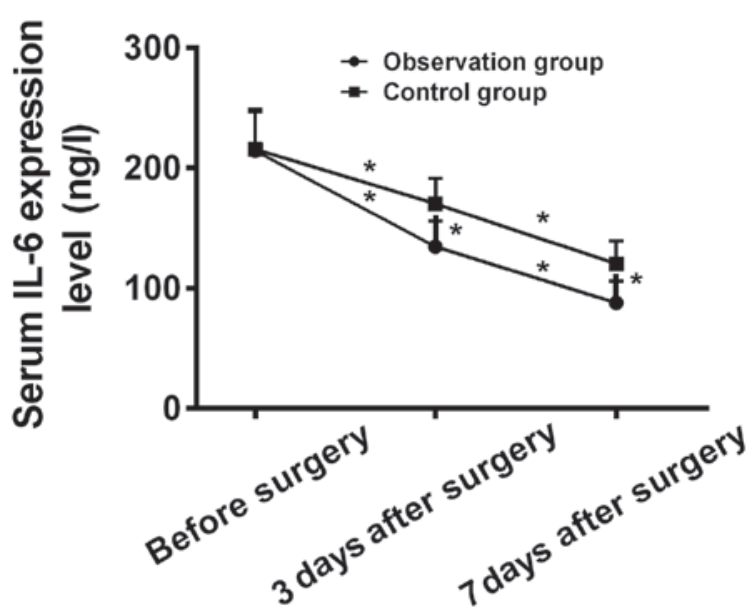

Figure 4. Expression level of IL-6 before and on the 3rd and 7th days after operation. Before treatment, there was no significant difference between the observation and control groups in the expression level of IL-6 ( $>00.05)$. On the 3rd and 7th days after operation, the expression levels in the two groups were significantly lower than those before treatment, and the expression level on the 7th day after treatment was significantly lower than that on the 3rd day after operation $\left({ }^{*} \mathrm{P}<0.05\right)$. On the 3 rd and 7 th days after operation, the expression levels in the observation group were significantly lower than those in the control group $\left({ }^{*} \mathrm{P}<0.05\right)$.

Perianal abscess is a common acute disease in proctology department. Infections or damage occurs in the anal gland or the hair follicle and the infections spread, eventually causing perianal abscess (12). Perianal abscess is difficult to treat and operation as its main treatment causes long wound healing time (13). In addition, patients with diabetes who are in a hyperglycemic state for a long time are prone to hypoproteinemia and decreased resistance. Moreover, hyperglycemia easily promotes the growth of bacteria and hinders wound surface healing (14). Therefore, it is urgent to better control the blood glucose of patients with diabetes complicated with 
perianal abscess in order to prevent poor operative efficacy and slow postoperative recovery caused by diabetes. Wound surface healing is a complex mechanism and it involves many cytokines among which inflammatory cytokine is an important one (15). A study shows that different administrations of insulin have significantly different effects on the blood glucose control of patients with diabetes, and insulin pumps are better than multiple subcutaneous injections in controlling hyperglycemia in critically ill patients, reducing inflammatory responses and improving short-term prognosis (16). However, there are currently few studies on differences in the blood glucose control of patients with diabetes complicated with perianal abscess between different administrations of insulin, as well as on the effects of the administration on the efficacy of radical operation of perianal abscess and postoperative recovery.

In this study, compared with the control group, patients in the observation group had significantly higher total effective rate $(\mathrm{P}<0.05)$, significantly shorter wound healing time and significantly lower growth score of the granulation tissue $(\mathrm{P}<0.05)$, and significantly lower FBG and 2hPBG levels after treatment $(\mathrm{P}<0.05)$. These findings suggest that insulin pumps for injection can regulate patients' blood glucose level and promote their postoperative recovery. In a previous study, insulin pumps slowly and continuously infused insulin through simulating physiological insulin secretion. This method not only replaces impaired insulin to work and quickly controls blood glucose, but also monitors patients' blood glucose level in real time and adjusts the dose and speed of pumping according to blood glucose changes, so it is more conducive to glucostasis (17). This also explains some conclusions of this study. According to a study, insulin pumps for blood glucose control improves the operative efficacy and promote the wound surface healing of patients with diabetes complicated with perianal abscess (18), which also confirms our conclusions. Among inflammatory cytokines, the expression of IL-6 is closely related to the intensity of inflammatory responses and the severity of tissue damage (19). In addition, the concentration of IL-6 can be increased by bacterial infections (20). TNF- $\alpha$ is the initiator of a chain of inflammatory responses and plays an important role in inflammatory responses. It promotes the release of IL- 6 and IL- 8 by promoting neutrophil aggregation, thus enhancing inflammatory responses in the body (21). According to comparison of the expression levels of TNF- $\alpha$ and IL-6, on the 3rd and 7th days after operation, the expression levels of TNF- $\alpha$ and IL- 6 in the observation and control groups were significantly lower than those before treatment, and the expression levels on the 7th day after treatment were significantly lower than those on the 3rd day after operation $(\mathrm{P}<0.05)$. On the 3rd and 7 th days after operation, the expression levels in the observation group were significantly lower than those in the control group $(\mathrm{P}<0.05)$. These findings indicate that insulin pumps for blood glucose control can reduce the expression of inflammatory cytokines and improve the inflammatory responses of patients after operation. A previous study shows that insulin inhibits the production of inflammatory cytokines (22). Therefore, insulin pumps are more effective for controlling the inflammation of patients with diabetes complicated with perianal abscess, possibly because its injection of insulin is more reasonable and controlled.
In summary, insulin pumps for injection during the perioperative period of patients with diabetes complicated with perianal abscess can better control the patients' blood glucose, improve the operative efficacy and promote the patients' postoperative healing. Moreover, continuous intravenous pumping of insulin is significantly better than traditional intermittent subcutaneous injection of it in controlling inflammation, so it is worthy of application. Insulin pumps are more effective than traditional intermittent subcutaneous injection, but the specific mechanisms promoting the operative efficacy and improving the inflammatory responses of patients with diabetes complicated with perianal abscess were not explored in this study. Therefore, the mechanisms still need to be investigated.

\section{Acknowledgements}

Not applicable.

\section{Funding}

No funding was received.

\section{Availability of data and materials}

The datasets used and/or analyzed during the present study are available from the corresponding author on reasonable request.

\section{Authors' contributions}

$\mathrm{ZZ}, \mathrm{HH}$ and $\mathrm{YH}$ were responsible for ELISA. WD helped with statistical analysis. ZZ wrote the manuscript. All the authors read and approved the final manuscript.

\section{Ethics approval and consent to participate}

The study was approved by the Ethics Committee of Jinhua Hospital of Zhejiang University (Jinhua, China). Patients who participated in this research had complete clinical data. The signed informed consents were obtained from the patients or the guardians.

\section{Patient consent for publication}

Not applicable.

\section{Competing interests}

The authors declare that they have no competing interests.

\section{References}

1. Dabelea D and Pettitt DJ: Intrauterine diabetic environment confers risks for type 2 diabetes mellitus and obesity in the offspring, in addition to genetic susceptibility. J Pediatr Endocrinol Metab 14: 1085-1091, 2001.

2. Kirkman MS, Mahmud H and Korytkowski MT: Intensive blood glucose control and vascular outcomes in patients with type 2 diabetes mellitus. Endocrinol Metab Clin North Am 47: 81-96, 2018.

3. Pasek RC and Gannon M: Advancements and challenges in generating accurate animal models of gestational diabetes mellitus. Am J Physiol Endocrinol Metab 305: E1327-E1338, 2013. 
4. Hanada M, Furuya T, Sugito K, Ohashi K, Ikeda T, Koshinaga T, Kawashima H, Inoue M, Hosoda T and Goto H: Evaluation of the efficacy of incision and drainage versus hainosankyuto treatment for perianal abscess in infants: A multicenter study. Surg Today 45: 1385-1389, 2015.

5. Hu Y: Advances in reducing cardiovascular risk in the management of patients with type 2 diabetes mellitus. Chronic Dis Transl Med 5: 25-36, 2019.

6. Murphy R, Tsai P, Jüllig M, Liu A, Plank L and Booth M Differential changes in gut microbiota after gastric bypass and sleeve gastrectomy bariatric surgery vary according to diabetes remission. Obes Surg 27: 917-925, 2017.

7. Fransgaard T, Thygesen LC and Gögenur I: Metformin increases overall survival in patients with diabetes undergoing surgery for colorectal cancer. Ann Surg Oncol 23: 1569-1575, 2016.

8. Pieber TR, Draeger E, Kristensen A and Grill V: Comparison of three multiple injection regimens for Type 1 diabetes: Morning plus dinner or bedtime administration of insulin detemir vs. morning plus bedtime NPH insulin. Diabet Med 22: 850-857, 2005.

9. Yki-Järvinen H and Kotronen A: Is there evidence to support use of premixed or prandial insulin regimens in insulin-naive or previously insulin-treated type 2 diabetic patients? Diabetes Care 36 (Suppl 2): S205-S211, 2013.

10. Ding L, Sousa KM, Jin L, Dong B, Kim BW, Ramirez R, Xiao Z, Gu Y, Yang Q, Wang J, et al: Vertical sleeve gastrectomy activates GPBAR-1/TGR5 to sustain weight loss, improve fatty liver, and remit insulin resistance in mice. Hepatology 64: 760-773, 2016.

11. Gijsbers L, Ding EL, Malik VS, de Goede J, Geleijnse JM and Soedamah-Muthu SS: Consumption of dairy foods and diabetes incidence: A dose-response meta-analysis of observational studies. Am J Clin Nutr 103: 1111-1124, 2016.

12. Machida T, Ohta S, Kakimoto Y, Toyoda A, Endou Y and Murakami O: A case of rectal cancer presenting with perianal abscess. Gan To Kagaku Ryoho 46: 88-90, 2019 (In Japanese).

13. Pearce L, Newton K, Smith SR, Barrow P, Smith J, Hancock L, Kirwan CC and Hill J; North West Research Collaborative: Multicentre observational study of outcomes after drainage of acute perianal abscess. Br J Surg 103: 1063-1068, 2016.

14. Mohammadi MH, Molavi B, Mohammadi S, Nikbakht M, Mohammadi AM, Mostafaei S, Norooznezhad AH, Ghorbani Abdegah A and Ghavamzadeh A: Evaluation of wound healing in diabetic foot ulcer using platelet-rich plasma gel: A single-arm clinical trial. Transfus Apheresis Sci 56: 160-164, 2017.
15. Xanthoulea S, Deliaert A, Romano A, Rensen SS, Buurman WA and van der Hulst RR: Nicotine effect on inflammatory and growth factor responses in murine cutaneous wound healing. Int Immunopharmacol 17: 1155-1164, 2013.

16. Huang W, Liu YS, Wang YJ, Long LM, Li X, Wang Y and Yang Y: A comparison of efficacy and safety in the treatment of hyperglycemia with continuous subcutaneous insulin with insulin pump or multiple insulin injections daily in critical elderly patients. Zhongguo Wei Zhong Bing Ji Jiu Yi Xue 20: 546-549, 2008 (In Chinese).

17. Conget I, Castaneda J, Petrovski G, Guerci B, Racault AS, Reznik Y, Cohen O, Runzis S, de Portu S and Aronson R; OpT2mise Study Group: The impact of insulin pump therapy on glycemic profiles in patients with type 2 diabetes: Data from the OpT2mise Study. Diabetes Technol Ther 18: 22-28, 2016.

18. Soedamah-Muthu SS and de Goede J: Dairy consumption and cardiometabolic diseases: Systematic review and updated metaanalyses of prospective cohort studies. Curr Nutr Rep 7: 171-182, 2018.

19. Lin ZQ, Dong YZ, Zhang XD, Wang T, Sun KL and Niu WY: Effect of interleukin-6 on gene expression of certain cytokines during wound healing process of mouse skin. Yi Chuan Xue Bao 32: 46-51, 2005 (In Chinese).

20. Zobel K, Martus P, Pletz MW, Ewig S, Prediger M, Welte T, Bühling F; CAPNETZ study group: Interleukin 6, lipopolysaccharide-binding protein and interleukin 10 in the prediction of risk and etiologic patterns in patients with community-acquired pneumonia: Results from the German competence network CAPNETZ. BMC Pulm Med 12: 1-10, 2012.

21. Borgatti M, Mazzitelli S, Breveglieri G, Gambari R and Nastruzzi C: Induction by TNF- $\alpha$ of IL- 6 and IL- 8 in cystic fibrosis bronchial IB3-1 epithelial cells encapsulated in alginate microbeads. J Biomed Biotechnol 2010: 907964, 2010.

22. Hanson MA and Gluckman PD: Early developmental conditioning of later health and disease: Physiology or pathophysiology? Physiol Rev 94: 1027-1076, 2014.

(i) $\Theta$ This work is licensed under a Creative Commons Attribution-NonCommercial-NoDerivatives 4.0 International (CC BY-NC-ND 4.0) License. 